\title{
COMPARATIVE STUDY BETWEEN AUSTIN MOORE PROSTHESIS AND BIPOLAR PROSTHESIS IN FRACTURE NECK OF FEMUR
}

\author{
Katragadda Sai Krishna1, D. Venkateswara Rao², G. Rajashekhar Reddy³, Anvesh Sangepu 4 \\ ${ }^{1}$ Senior Resident, Department of Orthopaedics, Siddhartha Medical College/Government General Hospital, Vijayawada. \\ ${ }^{2}$ Associate Professor, Department of Orthopaedics, Siddhartha Medical College/Government General Hospital, Vijayawada. \\ ${ }^{3}$ Consultant Surgeon, Department of Surgery, Royal Hospitals, Vijayawada. \\ ${ }^{4}$ Post Graduate, Department of Orthopaedics, Siddhartha Medical College/Government General Hospital, Vijayawada.
}

\section{ABSTRACT}

\section{BACKGROUND AND OBJECTIVE}

Femoral neck fractures have been considered 'Unsolvable fracture' in the olden era of orthopaedics due to high rate of associated complications, which include nonunion and avascular necrosis of the femoral head. Prosthetic replacement as a primary procedure eliminates osteonecrosis and non-union as complications of femoral fractures and also allows immediate weightbearing to return elderly patients to activity and help avoid complications of recumbency and inactivity. The decision to perform hemiarthroplasty using a unipolar or bipolar prosthesis remains controversial with proponents on either side. So in view of varied opinions, we desire to compare the efficiency of these two prosthesis, unipolar and bipolar prosthesis for the management of intracapsular fracture neck femur in elderly.

\section{METHODS}

One hundred and four patients above 60 years and an acute displaced fracture of the femoral neck were randomly allocated to treatment by either AMP or bipolar HA in the Department of Orthopaedics, AIMS, Government General Hospital, Vijayawada and Royal Hospital, Vijayawada between May 2011 and April 2015. The patients were summoned at 6 weeks, 12 weeks, 6 months and 1 year. Functional outcome was assessed and compared with Harris hip score and radiological parameters.

\section{RESULTS}

The two groups of patients with mean age of 70.4 in bipolar group and 69.1 in AMP group did not differ in their pre-injury characteristics (Sex, Fracture pattern, Comorbidity, Mode of injury and pre-injury ambulatory status) and perioperative parameters such as duration of operation, blood loss, hospital stay and mortality. The mean Harris hip score in Bipolar and AMP group was 90.03 and 84.4 respectively ( $\mathrm{p}=0.273$ ), range of motion was 234.6 and 221.3 with bipolar and AMP groups, respectively. Functional activities like use of public transport was better with bipolar group. Incidence of complications like superficial infection, haematoma and acetabular erosion was encountered in AMP group.

\section{CONCLUSION}

The use of a bipolar endoprosthesis in the management of displaced femoral neck fractures in the elderly was associated with better mean Harris hip score and incidence of complications was limited. Hence, bipolar would be a better option in elderly patients with fracture neck of femur. Moreover, the cost difference between AMP and bipolar prosthesis is not much in our country.

\section{KEYWORDS}

Unipolar- Bipolar- Hemiarthroplasty- Femoral Neck Fracture- AMP.

HOW TO CITE THIS ARTICLE: Katragadda Sai Krishna, D. Venkateswara Rao, G. Rajashekhar Reddy, Anvesh Sangepu. "Comparative Study Between Austin Moore Prosthesis and Bipolar Prosthesis in Fracture Neck of Femur." Journal of Evolution of Medical and Dental Sciences 2015; Vol. 4, Issue 101, December 17; Page: 16605-16610, D0I: 10.14260/jemds/2015/2477

\section{INTRODUCTION}

Hip fractures are devastating injuries that most often affect the elderly population and have a tremendous impact on the health care system and the society in general. ${ }^{1}$ It has been shown that hip fractures occur a decade earlier in Indians in comparison with Western Caucasian counterparts. ${ }^{1}$ The life expectancy at birth in 2010 was 65.1 years for Indian and as it shows an upward trend, increasing proportion of our population would face these problems. ${ }^{2}$

Financial or Other, Competing Interest: None.

Submission 27-11-2015, Peer Review 28-11-2015,

Acceptance 08-12-2015, Published 15-12-2015.

Corresponding Author:

Dr. D. Venkateswara Rao,

M. S. (Ortho), M. Ch. (Ortho), U. K,

$4^{\text {th }}$ Lane, Subbarao Colony, Flat No. 76,

H. No. 54/20/2-7A,

Opp: Chaitanya College Ladies Hostel,

Near Gurudwar Temple, Gurunanak Colony,

Vijayawada-520008.

E-mail:d_yenkee@yahoo.com

DOI:10.14260/jemds/2015/2477
Osteoporosis, comorbidities, increased incidence of trivial trauma increases the incidence and complicates the treatment of these fractures.

This high incidence is due to weak bones and increased incidence of trivial trauma.

People in this age group suffer from numerous illnesses that can aggravate the morbidity following fractures and complicate the treatment of these fractures. The treatment goal is to return the patient to his or her pre-morbid status of function. Increase in the average lifespan and improved medical facilities have greatly increased the incidence of these fractures. ${ }^{2}$ Moreover the awareness of osteoporosis in India is poor and the screening facilities for identifying is poor.

Management of femoral neck fractures in elderly patients has been controversial. Femoral neck fractures have been considered 'unsolvable fractures' in the older era of orthopaedics. $^{2}$ due to the high rate of associated complications, which include non-union, lack of rigid fixation and avascular necrosis of the femoral head among others. Presently, there are multiple surgical treatment options (Cannulated screws, dynamic hip screw systems, blade plates, 
hemi and total hip arthroplasty) available. Intracapsular extent of the fracture, tenuous blood supply to the femoral head going through the neck and difficulty in maintaining fracture reduction have been cited as reasons for failure of fixation.

Although treatment methods have been refined over the years, a consensus on the ideal treatment remains elusive. ${ }^{2}$ Important factors to consider in choosing any treatment modality are intrinsic, viz. patient age, general medical condition, type of fracture; and extrinsic, viz. availability of facilities and socio-economic status. Though non-operative treatment of these fractures has been documented. ${ }^{1}$ there are currently very few indications for the same (Being limited to terminally ill patients or those who are bedridden and nonambulatory). Surgical treatment has been established as the gold standard; however, the surgical option remains a dilemma. Open reduction and internal fixation has been shown to have a high rate of revision surgery due to nonunion and avascular necrosis hip replacement arthroplasty (Partial or total) is emerging as the most viable treatment option.

Prosthetic replacement allows immediate weight bearing to return elderly patients to activity and help avoid complications of recumbency and inactivity. When the concept of prosthetic replacement was first introduced, this perhaps was the most important advantage. As a primary procedure, prosthetic replacement eliminates osteonecrosis and nonunion as complications of femoral neck fractures. ${ }^{1}$ Prosthetic replacement of displaced femoral neck fractures reduces the incidence of reoperation compared with internal fixation. This argument applies only to elderly individuals with a limited life expectancy because the cumulative rate of reoperation for prosthetic replacement increases with time. ${ }^{3}$

The decision to perform hemiarthroplasty using a unipolar or bipolar prosthesis remains controversial with proponents on either side.

Advantages of the unipolar prosthesis include lower cost and no risk of polyethylene wear debris. Proposed advantages of the bipolar prosthesis include less acetabular wear and potentially less hip/groin pain. The choice between unipolar and bipolar prostheses is less clear. The main theoretical advantage of a bipolar over a unipolar prosthesis is the reduction of acetabular erosion due to movement taking place within the implant rather than between the head of the prosthesis and the acetabulum, although there is variation in the comparative distribution of the movement (Brueton et al. 1993). Movement within the prosthesis may also reduce the pain caused by the prosthesis moving against the acetabulum. ${ }^{3}$

\section{OBJECTIVES}

1. To compare the functional results obtained after hemiarthroplasties in intracapsular neck femur fractures in elderly people ( $\geq 60$ years) using non-cemented Austin Moore prosthesis and non-cemented non-modular bipolar prosthesis.

2. To study the associated complications in these cases.

- To find out the number of days hospital stay between the two prosthesis.

- To compare the recovery of physical, social and vocational independence and rehabilitation among Austin Moore and bipolar prosthesis.

- To compare the radiographic changes after hemiarthroplasty among Austin Moore and bipolar prosthesis.

\section{MATERIALS AND METHODS}

One hundred and four adult patients with fracture neck of femur treated at Dept. of Orthopaedics, Adichunchungiri institute of Medical Sciences, Government General Hospital, Vijayawada and Royal Hospital, Vijayawada between May 2011 and April 2015 were included in this study.

\section{Inclusion Criteria}

- Cases of fracture neck femur of age group above 60 years.

- All patients medically fit for surgery even with hypertension and diabetes mellitus.

- All types of fractures under Garden's classification are considered.

- Closed fracture.

- Fracture within 3 weeks.

\section{Exclusion Criteria}

- Seriously ill patients and Pts. not fit for surgery.

- Fracture due to tumor or any other pathological cause.

- Compound fractures.

- Other limb fractures and diseases.

- Neurovascular injuries.

In this study primary hemireplacement arthroplasty of hip is done in 104 cases of fracture neck of femur using bipolar prosthesis for 52 cases and Austin Moore's Prosthesis for 52 cases. The operative procedure is meticulously followed; the results are evaluated and compared.

\section{OBSERVATIONS AND RESULTS}

Out of 104 cases enrolled in the study, 6 cases from Group A were excluded from the study as they were lost to followup and 2 patient died due to chronic kidney disease. So at the end, 96 patients finished the study with 44 in AMP group and 52 in bipolar group. Of the 96 patients, 56 were females and 40 were males. The youngest patient in our series was 60 years old and the oldest patient was 91 years old. The average age of the patient in group A was 69.1 years and in group B was 70.4 years.

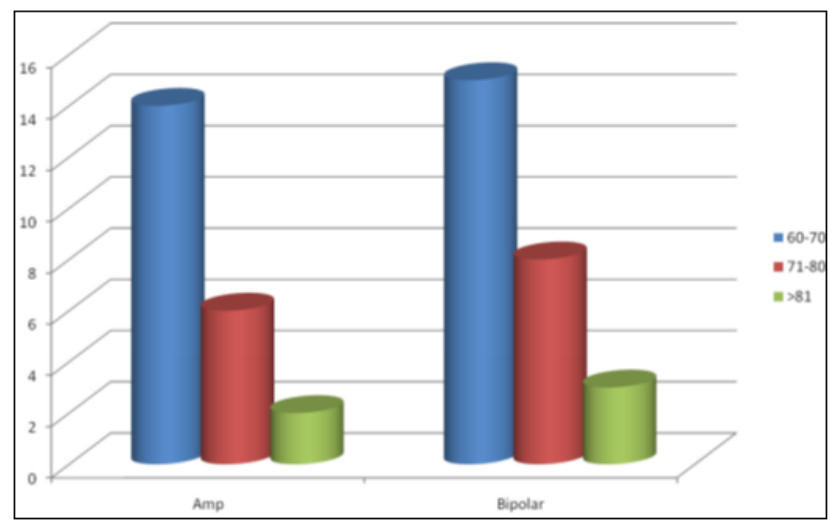

\section{AGE INCIDENCE}

The patients walked (Partial weightbearing) on an average 3.64 days after surgery 4 patients in group $A$ and 6 patients in group B developed superficial infection. All of them settled with IV antibiotics according to culture and sensitivity. Ten people developed haematoma probably due to early removal of drain. Deep seated infections were seen in 4 patients in Group A and 2 patients in Group B. Acetabular erosion was noted in 4 patients of Group A. There were no incidences of posterior dislocation or periprosthetic fractures in our study. 
This study signifies that with age group ranging 60-70, 92.9\% of the cases in Group A had excellent-to-good outcome, which was more compared to $86.7 \%$ of the cases in Group B, but the difference was insignificant. Age group ranging from $71-80,83.3 \%$ of the cases in Group A had excellent-to-good as their outcome, which was less compared to $100 \%$ results in Group B. Whereas age group belonging to $>81$ years, $50.0 \%$ of the cases in Group A had excellent-to-good outcome which was less compared to $100 \%$ in Group B, but the difference was not significant.

In our study, $27.2 \%$ of male patients in group A had excellent-to-good results which was less compared to $54.5 \%$ of females with excellent-to-good results, but the difference was statistically insignificant $(\mathrm{p}=0.2129)$. Similarly, in Group $\mathrm{B}$ both males and females had $96.7 \%$ excellent-to-good results $(p=0.549)$. In our study Group A patients were mobilized on an average of 4.04 post-operative day, whereas Group B were mobilized on 3.76 postop day.

\begin{tabular}{|c|c|c|c|c|}
\hline Age Group & Excellent & Good & Fair & Poor \\
\hline $60-70$ & $20(66.7 \%)$ & $6(20 \%)$ & $4(13.3 \%)$ & 0 \\
\hline $71-80$ & $10(62.5 \%)$ & $6(37.5 \%)$ & 0 & 0 \\
\hline$>81$ & $4(66.7 \%)$ & $2(33.3 \%)$ & 0 & 0 \\
\hline
\end{tabular}

ASSOCIATION OF AGE GROUP WITH OUTCOME - BIPOLAR

\begin{tabular}{|c|c|c|c|c|}
\hline Age Group & Excellent & Good & Fair & Poor \\
\hline $60-70$ & $14(50 \%)$ & $12(42.9 \%)$ & $2(7.1 \%)$ & 0 \\
\hline $71-80$ & $4(33.3 \%)$ & $6(50 \%)$ & $2(16.7 \%)$ & 0 \\
\hline$>81$ & 0 & $2(50 \%) 0$ & 0 & $2(50 \%)$ \\
\hline
\end{tabular}

\section{ASSOCIATION OF AGE GROUP WITH OUTCOME - AMP}

In our study $43 \mathrm{~mm}$ was the most commonly used prosthesis in Group A, whereas $41 \mathrm{~mm}$ was the most commonly used prosthesis in Group B.

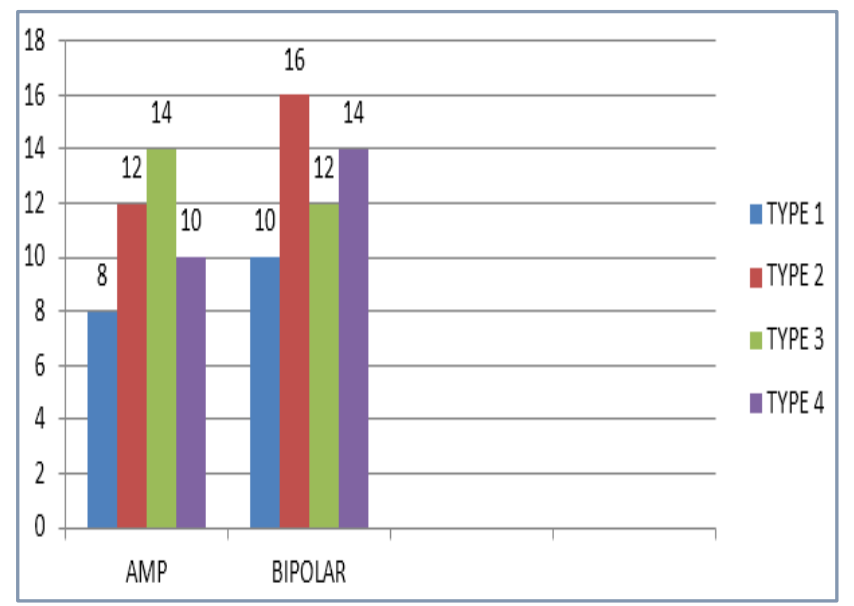

\section{GARDEN'S TYPE}

\section{ASSESSMENT OF FUNCTIONAL RESULTS}

The patients were enquired about the kind of pain they experienced during their daily activity and recorded according to the grades and scores; $73.1 \%$ of Bipolar Group and $40.1 \%$ of AMP Group had no pain. The difference in pain is statistically significant with a $\mathrm{p}$ value $=0.0095$.

All the patients in the study were enquired about the distance that they are able to walk and recorded and graded accordingly; $65.4 \%$ of bipolar group were able to walk unlimited and $45.5 \%$ of AMP group were able to walk unlimited. Difference in distribution of distance walked is statistically insignificant $(p=0.2592)$.

\begin{tabular}{|c|c|c|}
\hline PAIN & AMP & BIPOLAR \\
\hline $\begin{array}{c}\text { Marked pain with limitation of } \\
\text { activities }\end{array}$ & 0 & 0 \\
\hline Moderate & 0 & 0 \\
\hline Mild & $12(27.3 \%)$ & 0 \\
\hline Slight occasional & $14(31.8 \%)$ & $14(26.9 \%)$ \\
\hline None & $18(40.1 \%)$ & $38(73.1 \%)$ \\
\hline
\end{tabular}

\section{PAIN}

A 68.9\% of patients in Group AMP wore shoes with difficulty, whereas $53.9 \%$ of patients in Group Bipolar wore shoes with difficulty. Wear shoe and socks is easy in Group B, but the difference is statistically insignificant $(\mathrm{p}=0.0995)$.

A $69.23 \%$ of Bipolar group were able to use the public transport compared to only $54.5 \%$ of AMP group and the difference is statistically significant $\{p=0.0452$ (Fischer $t$ test)\}.

In our study, $50.0 \%$ of Bipolar group used no support for walking, whereas $31.8 \%$ of AMP group used no support for walking. None of the cases used two canes. The difference is statistically insignificant. ( $p=0.4000)$. In our study $59.09 \%$ patients in group A were able to sit for more than an hour which was less when compared to $80.8 \%$ in Group B, but the difference was statistically insignificant $\{p=0.1219$ (Fischer $t$ test)\}.

\begin{tabular}{|c|c|c|}
\hline Radiological & AMP & BIPOLAR \\
\hline Femoral stem loosening & 0 & 0 \\
\hline $\begin{array}{c}\text { Femoral stem-subsidence of } \\
\text { prosthesis }>5 m m\end{array}$ & 0 & 0 \\
\hline Sclerosis at the tip of prosthesis & 0 & 0 \\
\hline Acetabular erosion & $4(9.1 \%)$ & 0 \\
\hline Acetabular protrusion & 0 & 0 \\
\hline Heterotopic ossification & 0 & 0 \\
\hline Dislocation or subluxation & 0 & 0 \\
\hline
\end{tabular}

\section{RADIOLOGICAL CHANGES AT THE END OF 6 MONTHS}

\begin{tabular}{|c|c|c|}
\hline Complications & AMP & BIPOLAR \\
\hline Superficial infection & $4(9.1 \%)$ & $6(11.5 \%)$ \\
\hline Haematoma & $6(13.6 \%)$ & $4(7.7 \%)$ \\
\hline Gaping & 0 & 0 \\
\hline Post dislocation & 0 & 0 \\
\hline Prosthetic migration & 0 & 0 \\
\hline Acetabular erosion & $4(9.1 \%)$ & 0 \\
\hline Restricted ROM & $2(4.5 \%)$ & 0 \\
\hline Late infection & 0 & $4(7.7 \%)$ \\
\hline Sciatic nerve paresis & 0 & 0 \\
\hline Periprosthetic fracture & 0 & 0 \\
\hline Deep infection & $4(9.1 \%)$ & $2(3.8 \%)$ \\
\hline
\end{tabular}

\section{COMPLICATIONS}

\section{DISCUSSION}

The average age of the patient in group A was 69.1 years and in group B was 70.4 years. Majority of the patients were between $60-70$ years. The physiological age of our patients is more than the chronological age in all our patients. The elderly females are more prone to fracture neck of femur. $4,5,6$ Female preponderance has been reported in several series: Moore (1957): 62.5\%, Campbell (1960): 80.9\%; Cone (1963): 73.6\%; Anderson and Neilson (1972): 85\%; Sikroski and Barrington (1981): 66.7\%; Arwade (1987): 68.3\%, John E. Kenzora (1998): 77.4\%; Carl Johan Hedbeck (2010): 76\%; Bhushan MS (2011): 78.5\%. Male preponderance is reported in few series: D'Acry and Devas (1976).7 91.4\%; Mukherjee and Puri (1986): 58.3\%; Amte and Sanchetti (1987): 55\%; Bavadekar and Manelkar (1987): 60.9\%. Male femoral neck 
fracture patients are in general younger than female patients. In our study female preponderance was $65.8 \% .8,9$

Boyd and Salvatore (1964) reported 55\% fractures on left side. D'Acry and Devas. ${ }^{6}$ (1976) similarly found $55.4 \%$ fracture in left hip of their patients. Left sided hip was fractured in $60.9 \%$ of our cases; $65 \%$ of bipolar group and $57.1 \%$ of AMP were left sided fracture. In our study we reported 63.6\% right sided fractures in Group A and 53.8\% in group B. Depending on the anteroposterior view in internal rotation, the fracture pattern is classified among Garden type 1 to 4 . Majority of the fractures in our study belong to types 2 and 3, 59.1\% in Group A and 53.8\% in Group B. Kulkarni GS (1987) had grouped type III and type IV into one group of displaced fractures and reported it in $82.5 \%$ of his patients.

Sanchetti et al. (1987) reported 30\% Garden type III and $22.5 \%$ Garden type IV in a series distributed between 20 to 80 years of age. Mukherjee and Puri (1986) had 85\% patients of Garden type III and IV fractures.

The types of displacement (Gardens III and IV) are not taken as the criteria to choose the procedure for the management of fracture neck of the femur. The age of the patient [Saraf and Saxena. ${ }^{9}$ (1978), Mukherjee and Puri. ${ }^{10}$ (1986), Arwade. ${ }^{11}$ 1987) and time since fracture [Boyd and Salvatore. ${ }^{12}$ (1964), Salvatti et al. ${ }^{13}$ (1973), Sikroski and Barrington (1981), Kulkarni GS (1987). ${ }^{13}$ are taken into consideration while selecting hemiarthroplasty for the management of fracture neck of femur.

A $100.0 \%$ of our patients had trivial trauma (Self-fall). This is in accordance with majority of the series reported[Gyepes (1962), Solomon (1968), Evarts (1973), Fielding (1974), Ingalhalikar (1987), Seth (1987) etc.].14,15,16 Stevens et al. (1962), Scott and Gray (1980), Urovitz et al. (1977), Colonel M.K. Seth (1987) and several other authorities believe that the intracapsular fracture are stress fractures through pathological bone secondary to osteoporosis or osteomalacia. We had no operative deaths in our series. Totally 2 patients $(4.5 \%)$ expired during the followup in our series from Group A. The death was due to Chronic Kidney disease. Nather et al. reported on a series of 110 patients with mean age of 78 years, treated by unipolar, found a one year mortality of $15 \% .{ }^{17} \mathrm{Su}$ et al. reviewed the in-hospital mortality rates of patient's age greater than 65 years. They reported greater than 50,000 patients and found overall mortality rate of $5.6 \% .{ }^{18}$ Clyer and Bruckner found mortality rates of $22 \%, 63 \%$ and $81 \%$ at 1,5 and 10 years after hemiarthroplasty using AMP. 19

In our series all the patients were discharged after the patient is trained for active mobilization and when fit were for discharged. Most of our patients, $57.7 \%$ of Bipolar and $54.5 \%$ of AMP group stayed between $10-15$ days, but $19.2 \%$ of Bipolar were discharged within 10 days because of their active recovery when compared to $13.6 \%$ in AMP group. Post-operative stay is statistically similar. Cadler.20 (1999) found that the length of stay in hospital was not significantly different for the two groups $(\mathrm{p}=0.40)$; patients with the Monk prosthesis spent a median of 17 days in hospital (13 to 22), while those with a Thompson prosthesis had a median hospital stay of 18 days (13 to 23). According to Cornell.21 there were no differences in the postoperative complication rates or lengths of hospitalization were seen between the two groups.

All our patients were mobilized as early as possible depending on patient compliance. Most of the patients were mobilized within 3 days in both groups, $61.5 \%$ in bipolar and $63.6 \%$ in AMP. Four patients (9\%) from AMP group when followed up to one year presented with painful hip and acetabular erosion. Shortening of $2 \mathrm{~cm}$ was noted and gross restricted range of motion was observed, thereby limiting his functional activity. It was classified as grade 2 in both cases according to Baker. Whittaker et al. reported in a series of 160 hemiarthroplasty cases, rate of joint spacing in 5-year study was $64 \%$ with AMP prosthesis. ${ }^{22}$ D_Arcy and Devas reported experience in unipolar prosthesis in a study of 361 cases, acetabular erosion of $11 \%$ and posterior dislocation in $2 \% .{ }^{6}$ The theoretical advantage of the bipolar design was to dissipate the joint forces through the inner bearing surfaces, thereby decreasing the rate of superior acetabular erosion. Cadler described acetabular erosion after hip hemiarthroplasty is a longer-term problem in younger patients. There were three cases in the unipolar group and none in the bipolar, which may demonstrate the theoretical benefit of the bipolar prosthesis, although the differences were not statistically significant. ${ }^{21}$

Acetabular erosion was graded according to the criteria of Baker et al. as grade 0 (No erosion), grade 1 (Narrowing of articular cartilage, no bone erosion), grade 2 (Acetabular bone erosion and early migration), and grade 3 (Protrusion acetabuli). ${ }^{23}$ In our study, we had 10 cases of superficial infection, $6(11.5 \%)$ in bipolar and $4(9 \%)$ in AMP group. It was managed conservatively with IV antibiotics based on the culture growth; $6(13.6 \%)$ patients in AMP Group and 4(7.6\%) patients in Bipolar Group developed Haematoma. Some of these patients had incidents of accidental drain removal. We believe that proper suturing of drain is important for preventing haematoma. We had 6 cases of Deep infection $4(9 \%)$ in Group A and $2(3.8 \%)$ in Group B probably due to poor hygiene and uncontrolled diabetes. Increased incidence of infection has been reported with using posterior Moore's approach for hemiarthroplasty. Reported incidences of superficial infection after primary prosthetic arthroplasty include Salvatti et al. (1973) -8.3\%; Salvatti et al. (1973) 8.3\%; Saraf and Saxena (1978) 3.7\%; Mukherjee and Puri (1986) 3\%; Bavadekar and Manelkar (1987) 7.6\% and Jack and Moshein (1990) 2.3\%. The infection was no ways related to the prosthesis. Overall, the incidence of complication rate is more noted with AMP group.

Lanceford (1965) felt that the pain following hemiarthroplasty should not be the cause for condemning the procedure. He listed following causes for pain: Infection, improper prosthetic seating, metallic corrosion and tissue reaction, improper sized femoral head, contractures, periarticular ossification, toggle or acetabular wandering and redundant ligamentum teres. ${ }^{24}$ In our series, $73.1 \%$ of bipolar and $40.1 \%$ of AMP had no pain. Distribution of pain is less in Bipolar group and the difference is statistically significant $p=0.009$. Studies showing $\%$ with no pain. Gingras et al. studied cemented unipolar endoprosthesis for femoral neck fracture over an average follow-up period of 17 months. Ninety-two percent had poor slight pain, but $8 \%$ had evidence of protrusion. ${ }^{25}$

Limping is a common consequence of hemiarthroplasty in adults. Exact cause cannot be attributed to this. Alteration in the abductor mechanism due to excision of little more neck is the most probable cause [Saraf and Saxena, (1978); Hinchey and Day (1964)]. ${ }^{26}$ All the patients were asked to use a cane on the sound side regularly. This decreases load on the prosthetic head. Once the patient got enough endurance, they were advised to discard the cane. Saraf and Saxena (1978) reported $52.7 \%$ patients using cane regularly, $23.1 \%$ occasionally and $21.8 \%$ were not using it; $80.8 \%$ of bipolar group and $59.09 \%$ of AMP group were able to sit in a chair comfortable for more than an hour. Statistically both the groups are similar with $p$ value $=0.1219$. Mean ROM of bipolar was 234.6 compared to 221.3 in AMP. Cornell et al. ${ }^{27}$ (1998) who reported that patients with bipolar prosthesis did better on walk tests and had better range of motion at 6 months. ${ }^{21} \mathrm{~A}$ $65.4 \%$ of bipolar were able to walk unlimited compared to only $45.5 \%$ of AMP. There was no statistical difference 
between the two groups ( $\mathrm{p}=0.2592)$. Bhushan M Sabnis, Ivan J Brenkel 100 reported 14\% unipolar walking unaided compared to $54 \%$ of bipolar walking alone outside.

All the cases in our series were assessed according to Harris Hip Score and graded accordingly as Excellent, Good, Fair, Poor and Failure. We got $65.3 \%$ excellent result with Bipolar group and $40.9 \%$ with AMP group. The mean HHS was 90.03 in Bipolar and 84.4 in AMP group. Distribution of result is statistically similar in both groups $(\mathrm{p}=0.3283)$, but the mean score is statistically more associated with patients with bipolar prosthesis. Yamagata et al., in their classical study of reviewed 1001 cases of hip hemiarthroplasty, there were 682 unipolar and 319 bipolar cases.

Patients undergoing bipolar exhibited higher hip score and lower acetabular erosion rates compared to unipolar patients. ${ }^{28}$ Bochner et al. reported their experience with bipolar arthroplasties in a consecutive series of 120 hemiarthroplasties.

In this group, 90 patients were followed for at least 2 years with $91 \%$ being pain free and $92 \%$ demonstrating satisfactory power and motion. ${ }^{29}$

Hence, compared to previous studies, in our study there appears to be a significant difference between the two groups functionally; better function with range of movement, use of public transport and pain, are associated better with bipolar group. And also mean HHS is better with bipolar group.

\section{CONCLUSION}

- Primary Hemiarthroplasty is an efficient way for treatment of displaced intracapsular neck of femur in elderly patients. The success of hemiarthroplasty depends on proper pre-operative planning, aseptic precautions, co-morbid conditions and attention to surgical details.

- At the end of 1 year, the percentage of patients achieving good-to-excellent outcome with Bipolar prosthesis were more than those with AMP, though the difference was not statistically significant.

- After the end of 1 year, mean Harris Hip score was 84.4 in Group A which was less when compared to 90.03 of Group B.

- Incidence of complications also were more in Group A when compared to Group B, though the difference was not statistically significant.

- The mortality, postop stay and time of mobilisation also was similar between the two groups.

- There was no significant radiological difference between the two groups.

- Comparing the functional assessment, all the parameters were similar except for use of public transport being better with bipolar group.

- Pain also was better in Group B when compared to Group A.

- Which type of hemiarthroplasty should we select for the most elderly patients with displaced fractures of the femoral neck? Based on the results of our study and previous ones, there appears to be a statistical difference between the two groups, that is bipolar being better in functional aspects. On the other hand, the results of our study showed that incidence of complication were lower after the bipolar $\mathrm{HA}$, which in turn may indicate an advantage in the longer term.

- Some of the Western literature report that disadvantage of Bipolar being a higher cost, but it was not considered in our institution, as there is not much cost difference between the two prosthesis.
- Limitation of the study is that the period of study is less, and sample is small number. Although an unbiased observer assessed all clinical variables except hip motion, this observer was not blinded to the type of surgical intervention, which may add a risk of bias.

\section{REFERENCES}

1. Campbell's Operative Orthopaedics 11th edition. Hip Fractures. 2007;3274.

2. TR Bandgar. Vitamin D and Hip Fractures: Indian Scenario. Journal of Association of Physicians of India. September 2010;58.

3. SJ Calder, GH Anderson, C Jagger, et al. J Bone Joint Surg [Br] 1996;78-B:391-4.

4. Butler $M$, Norton $R$, Lee-Joe $T$, et al. The risk of hip fracture in older people from private homes and institutions. Age ageing 1996;25:381-5.

5. Fisher ES, Baron JA, Malenka OJ, Barrett JA, Kniffin WD, Whaley FS, et al. Hip fracture incidence and mortality in New England. Epidem 1991;2:116-22.

6. Hinton RY, Smith GS. The association of age, race and sex with the location of proximal femoral fractures in the elderly. J Bone Joint Surg 1993;75A:752-9.

7. Kwok DC, Cruess RL. A retrospective study of more and Thompson hemiarthroplasty. Clin Oth Rel RES 1982;169:179-85.

8. Huuskonen J, Kroger H, Arnala I, et al. Characteristics of male hip fracture patients. Ann ChirGyn 1999;88:48-53.

9. Saxena PS and Saraf JK Moore. Prosthesis in fracture neck of femur. Indian Journal of Orthopaedics 1978; Vol 12:138-45.

10. Mukherjee DL (Col), Maj. Gen. HC Puri. Early hemiarthroplasty for fresh fractures of the neck of the femur in geriatric patients. Indian Journal of Surgery 1986; Vol. 48:77-80.

11. Arwade DJ. A review of internal fixation and prosthetic replacement for fresh fractures of the femoral neck. CliniOrthop India 1987;1:77-82.

12. Boyd HB and Salvatore JE. Acute fractures of the femoral neck: Internal fixation or Prosthesis? JBJS 1964;46A:1066-8.

13. Kulkarni GS. Pathology of fracture neck of the femur. Clinical Orthopaedics of India 1987;1:92-6.

14. Evarts CM. Endoprosthesis as the primary treatment of femoral neck fractures. Clinc Orthop 1973;92:69-76.

15. Ingalhalikar VT, Shekar Kumta. Fracture neck femur anatomical and biomechanical aspects. Clinc Orthop India 1987.

16. Seth MK (Col). Stress fractures of the neck of femur. Clinical Orthropaedics India 1987;1:105-9.

17. Nather A, Seow CS, Iau P, et al. Morbidity and mortality for elderly patients with fractured neck of femur treated by hemiarthroplasty. Injury, 1995;26:187-90.

18. Su H, Aharonoff GB, Hiebert R, et al. In hospital mortality after femoral neck fracture: do internal fixation and hemiarthroplasty differ? Am J Orthop 2003;32:151-5.

19. Clyer M, Brucknerj. The outcome of Austin-Moore prosthesis for fracture of the femur neck, Am J Orthop 1997;26:681-4.

20. Nottage WM, McMaster WC. Comparison of bipolar implants with fixed neck prosthesis in femoral neck fractures. ClinOrthop 1990;251.

21. Calder SJ, Anderson GH, Jagger C, et al. Unipolar or bipolar prosthesis for displaced intracapsular hip fracture in octogenarians: a randomised prospective study J Bone Joint Surg Br 1996;78:391-4. 
22. Whittaker RP, Aberhaus MM, Scholl HW, et al. Fifteen years' experience with metallic end prosthesis replacement of the femoral head for femoral neck fractures. J Trauma 1972;12:799-806.

23. Baker RP, Squires B, Gargan MF, et al. Total hip arthroplasty and hemiarthroplasty in mobile, independent patients with a displaced intracapsular fracture of the femoral neck. A randomized, controlled trial. J Bone Joint Surg Am 88:2583-9.

24. Lance Ford EM. Use of Moore self-locking Vitallium prosthesis in acute fractures of the femoral neck. JBJS 1965;47A:832-841.

25. Gingras MB, Clarke J, Evans CM. Prosthetic replacement in femoral neck fractures. Clin Orthop 1980;152:47.
26. Hinchey and Day. Primary prosthetic replacement in fresh femoral neck fractures. JBJS 1960;42B:633-40.

27. Bhushan M Sabnis, Ivan J Brenkel. Unipolar versus bipolar uncemented hemiarthroplasty for elderly patients with displaced intracapsular femoral neck fractures: Journal of Orthopaedic Surgery 2011;19(1):8-12.

28. Bochner RM, Pellicci PM, Lyden JP. Bipolar hemiarthroplasty for fracture of the femoral neck. Clinical review with special emphasis on prosthetic motion. J Bone Joint Surgery Am 1988;70:1001-10.

29. Lestrange NR. Bipolar arthroplasty for 496 hip fractures. Clin Orthop 1990;251:7-19. 